\title{
LEED STUDIES OF LEAD ON COPPER (100)
}

\author{
W. HOESLER and W. MORITZ \\ Institut für Kristallographie und Mineralogie, SFB 128. Unversität München. Theresienstrasse 4I, \\ D. 8000 München, Fed. Rep. of Germany
}

Received 13 September 1981; accepted for publication 15 October 1981

Lead layers on copper (100) at coverages from 0.5 to 0.6 were investigated by quantitative LEED and AES measurements and dynamical LEED calculations. Comparisons of intensity measurements with dynamical calculations for the $(\sqrt{2} \times \sqrt{2}) \mathrm{R} 45^{\circ}$ structure show that the $\mathrm{Pb}$ atoms are adsorbed in hollow sites. The $\mathrm{Cu}-\mathrm{Pb}$ distance is close to the sum of both metallic radii. The dense lead monolayer at a coverage of 0.6 exhibits a $c(5 \sqrt{2} \times \sqrt{2}) \mathrm{R} 45^{\circ}$ superstructure with $\mathrm{cmm}$ symmetry of the diffraction pattern. Calculations for five models were performed under variation of several geometric parameters. Most analogies were found for regular arrangements of distorted $(\sqrt{2} \times \sqrt{2}) \mathrm{R} 45^{\circ}$ domains, separated by antiphase boundaries. This result is in consistency with observations of poorly ordered $(\sqrt{2} \times \sqrt{2}) \mathrm{R} 45^{\circ}$ antiphase domains at intermediate coverages.

\section{Introduction}

Several dense and nearly dense monolayers of metals on fourfold substrates exhibit the same types of superstructure. These structures are characterized by centered rectangular unit cells and can be described as $\mathrm{c}(x \sqrt{2} \times \sqrt{2}) \mathrm{R} 45^{\circ}$, with $x$ as odd integer between 3 and 9 [1]. For example lead on gold (100) forms a $\mathrm{c}(3 \sqrt{2} \times \sqrt{2}) \mathrm{R} 45^{\circ}$ [2] and bismuth on copper $(100)$ a $\mathrm{c}(9 \sqrt{2} \times \sqrt{2}) \mathrm{R} 45^{\circ}$ structure [3]. The lateral adsorbate spacing in one direction is identical to the spacing of the $(\sqrt{2} \times \sqrt{2}) \mathrm{R} 45^{\circ}$ structure exhibited by all these systems at lower coverages. In contrast to this, the other cell vector is essentially elongated, obviously caused by one-dimensional compression and shearing of chains of adsorbate atoms. Suggestions have been made to explain these structures by pseudo-hexagonal arrangements [2-5], and contrary to this by one-dimensional series of $(\sqrt{2} \times \sqrt{2}) \mathrm{R} 45^{\circ}$ antiphase domains [6,1]. Both models cannot be distinguished by simple considerations of the diffraction pattern, but require an intensity analysis by dynamical LEED calculations.

The two models represent different mechanisms of adsorption and reconstruction of the topmost layer; the first assumes nearly free atoms occupying arbitrary sites on the substrate, the second considers them trapped in fixed sites. Thus a solution of these structures will outline the influence of lateral potential variation on structure formation.

0039-6028/82/0000-0000/\$02.75 (1) 1982 North-Holland 
Lead on copper (100) forms a $c(5 \sqrt{2} \times \sqrt{2}) \mathrm{R} 45^{\circ}$ superstructure [5]. The applicability of dynamical LEED calculations is supported by the good agreement of calculations with experiments on clean copper (100) [7]. The size of the unit cell still permits a theoretical treatment, however the energy range is limited. The anomalous low chemical reactivity of the complete monolayer with oxygen [8], as well as the barrier against formation of a second layer [9] draws additional interest in the structure of this system.

\section{Experimental}

Experiments were performed in a standard UHV chamber at pressures well below $10^{-10}$ Torr. The crystal was cleaned as usual by cycles of ion bombardment and heating. Lead was evaporated from an rf-heated and outgassed $5.5 \mathrm{~N}$ source. After preparation no impurities on the substrate and in the adsorbed layers could be detected by AES. The coverage was determined by evaluating the heights of the 90 and $94 \mathrm{eV}$ lead Auger peaks.

Intensities were measured by a movable Faraday cup. Two-axis motion of the cup, setting of electron energy and data sampling were computer controlled. Preliminary measurements on copper (100) gave excellent agreement with theoretical curves and with the results of Noonan and Davies [7].

\section{Details of calculations}

LEED intensity calculations were performed using the layer doubling method for wave propagation between planes and the matrix inversion method for intra-layer scattering [10]. Up to eight spin averaged phase shifts were used. The atomic charge densities were calculated for the free atom using a modified Slater exchange term [11], and the phase shifts were obtained using the bulk structure in calculating the overlap of charge densities. The same program [12] has been used for lead and copper. For clean copper the real part for this set of phase shifts was found to be $V_{0}=8 \mathrm{eV}$; the imaginary part was set to $V_{\mathrm{i}}=0.85\left(E+V_{0}\right)^{1 / 3} \mathrm{eV}$ to compare the results with those of Noonan and Davis [7]. The same values as for clean copper have been taken for the real and imaginary part of the inner potential within the lead overlayer.

The Debye temperature for copper was set to $330 \mathrm{~K}$ and for lead to $70 \mathrm{~K}$. In case of the $(\sqrt{2} \times \sqrt{2}) R 45^{\circ}$ structure the Debye temperature of lead was varied between 110 and $40 \mathrm{~K}$. However, the influence on the $I-V$ cuves was not significant enough to derive an exact surface Debye temperature for the lead layer.

A maximum of 24 symmetrically independent beams were included in the calculations for the $(\sqrt{2} \times \sqrt{2}) \mathrm{R} 45^{\circ}$ models with $4 \mathrm{~mm}$ symmetry, correspond- 
ing to 40 beams for the bridge site model with twofold axis. In case of the $\mathrm{c}(5 \sqrt{2} \times \sqrt{2}) \mathrm{R} 45^{\circ}$ structure only one mirror plane could be used for the superstructure beams, though all models considered here show cmm symmetry. This restriction together with a total number of 80 beams limited the energy range to about $160 \mathrm{eV}$.

\section{Results}

Sharp reflections of the $(\sqrt{2} \times \sqrt{2}) \mathrm{R} 45^{\circ}$ structure are visible at a well defined and narrow coverage range around 0.5 . At lower coverages $(2 \sqrt{2} \times$ $2 \sqrt{2}) \mathrm{R} 45^{\circ}$ reflections occur and at slightly higher coverages the half order beams become diffuse. Further adsorption results in splitting of the superstructure beams and finally at a coverage of 0.6 in formation of the $\mathrm{c}(5 \sqrt{2} \times \sqrt{2}) \mathrm{R} 45^{\circ}$ structure, which again exhibits sharp reflections (fig. 1). Further evaporation neither changes the Auger peak heights nor the LEED diffraction pattern.

\section{1. $(\sqrt{2} \times \sqrt{2}) R 45^{\circ}$ structure}

The intensities of seven beams were measured at normal incidence and at a crystal temperature of $160 \mathrm{~K} . I-V$ curves were obtained by averaging $I(h k)$
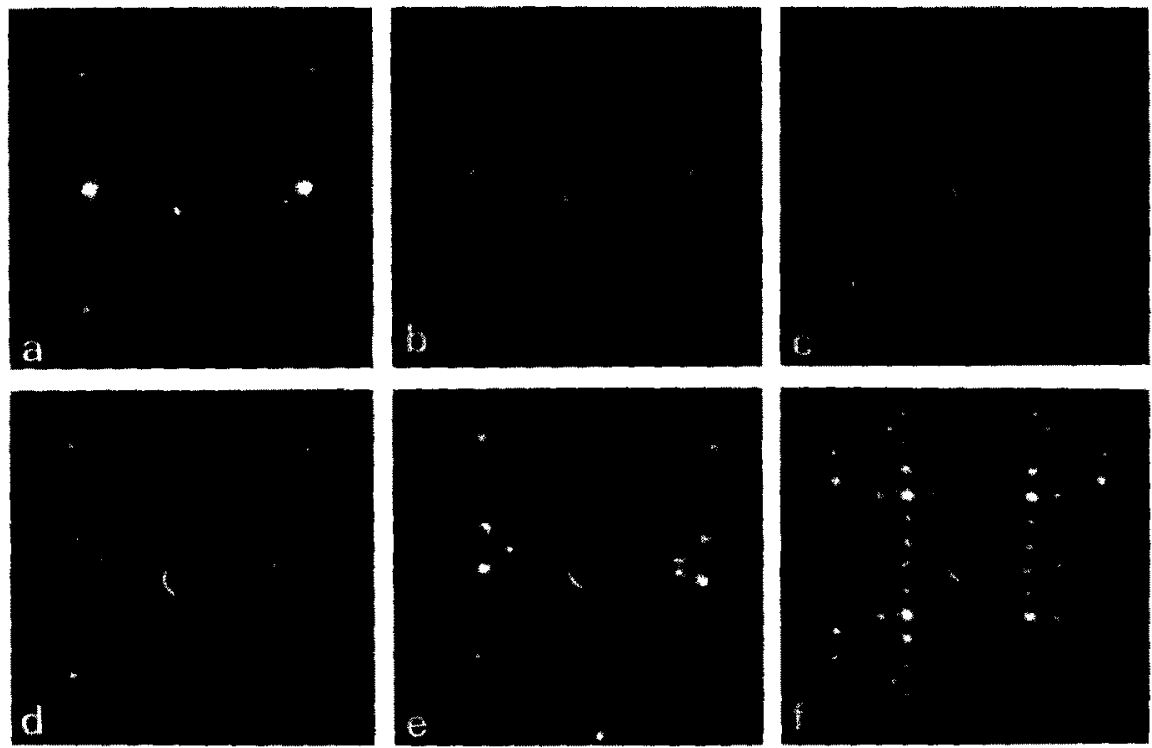

Fig. 1. LEED patterns of $\mathrm{Cu}(100)-\mathrm{Pb}$ at coverages from 0.5 to 0.6 . The energy of the primary beam is $48 \mathrm{eV}$. (a) $(\sqrt{2} \times \sqrt{2}) \mathrm{R} 45^{\circ}$ structure at a coverage of 0.5 . (b), (c), (d) Change during increase in coverage. (e) a $5 \sqrt{2} \times \sqrt{2}) \mathrm{R} 45^{\circ}$ structure at a coverage of 0.6 . (f) Same structure as (e) at $155 \mathrm{eV}$. 

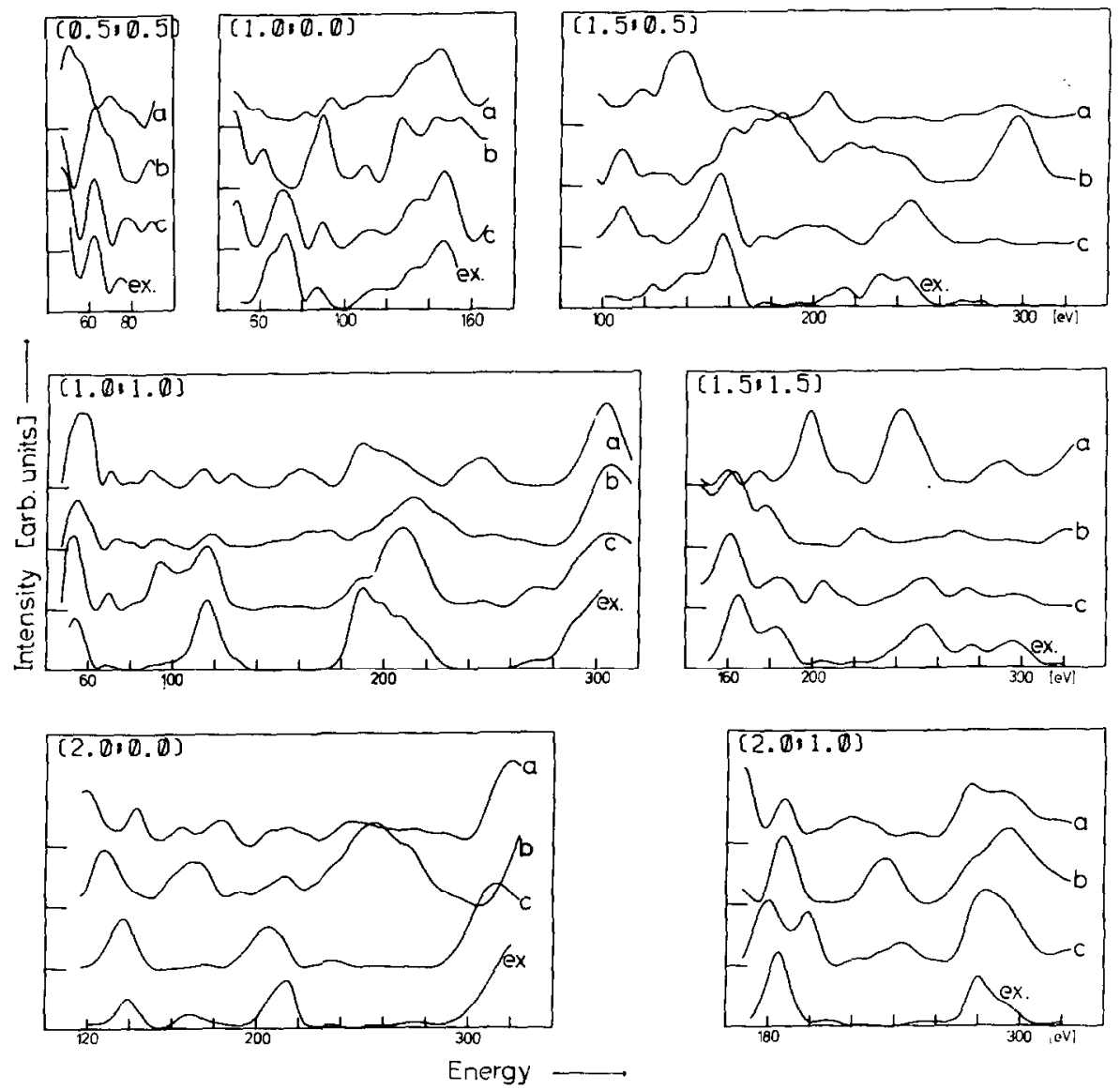

Fig. 2. Comparison between theoretical and experimental spectra of the $(\sqrt{2} \times \sqrt{2}) \mathbf{R} 45^{\circ}$ structure at normal incidence. Theoretical curves for three different adsorption sites of the $\mathrm{Pb}$ atoms at layer spacings $\mathrm{d} z$; bridge site $\mathrm{d} z=2.9 \AA$ (a), top site $\mathrm{d} z=3.0 \AA$ (b), hollow site $\mathrm{d} z=2.4 \AA$ (c): crystal temperature $T=160 \mathrm{~K}$; Debye temperatures $\Theta_{\mathrm{Cu}}=330 \mathrm{~K}, \Theta_{\mathrm{Ph}}=70 \mathrm{~K}$.

and $I(\bar{h} \bar{k})$ and subtracting the background. The limited moveability of the Faraday cup allowed only the measurement of these two out of four or eight degenerate beams.

The $(\sqrt{2} \times \sqrt{2}) \mathrm{R} 45^{\circ}$ structure is described by alternate occupation of adsorption sites on the substrate surface. The $\mathrm{Pb}-\mathrm{Pb}$ distance of this structure is $3.62 \AA$ and only slightly larger than the value of bulk lead.

Three adsorption sites of high symmetry are possible, hollow, top and bridge site. Comparison of experimental data with calculations for the three sites is presented in fig. 2. Best agreement was found for the hollow site model at a layer distance of $2.40 \pm 0.05 \AA$. The minimum average $r$-factors [13] are 0.17 for hollow site, 0.22 for top site, and 0.34 for bridge site. Visual 
comparison of the $I-V$ curves leads to a clearer preferences for the hollow site model, than expected by consideration of the small differences in $r$-factors. This is due to the $r$-factor, defined by Zanazzi and Jona [13], which compares the first and second derivatives but neglects the relative peak heights. For comparison of the different models a $\mathrm{Pb}$ Debye temperature of $70 \mathrm{~K}$ has been used, corresponding to the bulk value. Debye temperature variation for the hollow site model leads to a faint minimum of $r=0.16$ at $\Theta_{\mathrm{pb}}=50 \mathrm{~K}$.

The top layer spacing of $2.4 \AA$ corresponds to a $\mathrm{Cu}-\mathrm{Pb}$ distance of $3.0 \AA$ which is nearly identical to the sum of both metallic radii.

\section{2. $c(5 \sqrt{2} \times \sqrt{2}) R 45^{\circ}$ structure}

I- $V$ curves were taken for 25 beams of the $\mathrm{c}(5 \sqrt{2} \times \sqrt{2}) \mathrm{R} 45^{\circ}$ structure in the same way as described above. Only 14 of them could be compared with model calculations, since the computable energy range was limited to $160 \mathrm{eV}$.
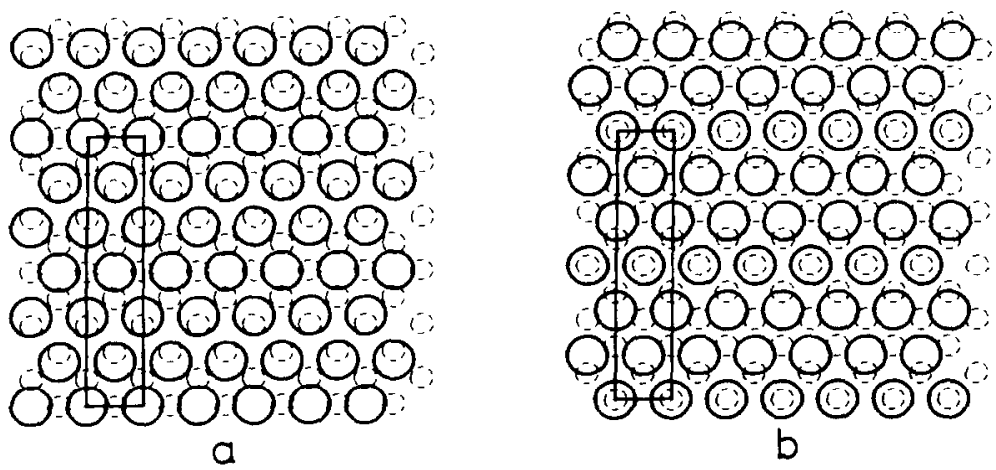

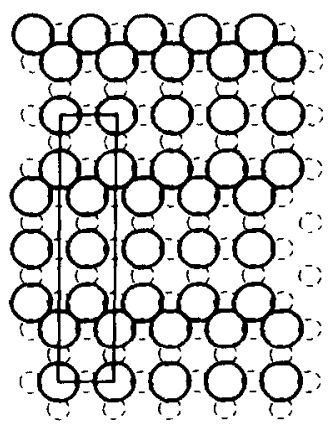

C

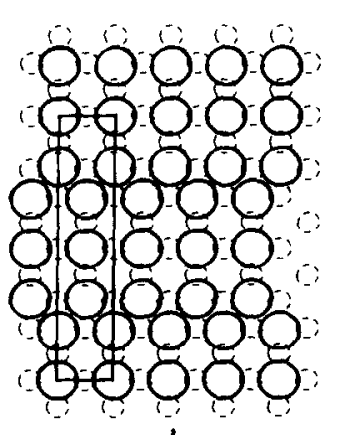

d

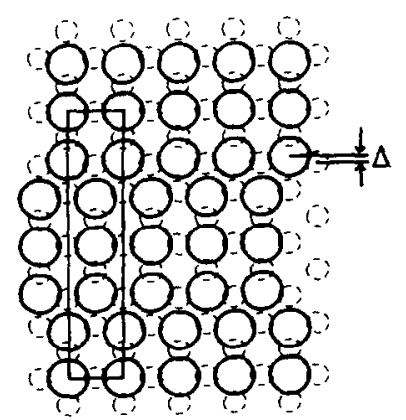

e

Fig. 3. Models for the $c(5 \sqrt{2} \times \sqrt{2})$ R $45^{\circ}$ structure used for the calculated spectra in fig. 4 : pseudo-hexagonal structures with one atom in hollow site (a) and top site (b); antiphase domains of the $(\sqrt{2} \times \sqrt{2}) \mathrm{R} 45^{\circ}$ structure with all atoms in hollow sites (c); $\mathrm{Pb}$ atoms shifted from hollow site by $\Delta=0.2 \AA$ (d) and $0.4 \AA$ (e). 
Two models have been proposed in early studies of this system. Henrion and $\mathrm{Rhead}$ [5] proposed a pseudo-hexagonal layer, whereas Biberian and Huber [1] preferred the high symmetry model introduced by Huber and Oudar [6]. The latter consists of $(\sqrt{2} \times \sqrt{2}) \mathrm{R} 45^{\circ}$ regions, regularly separated by antiphase boundaries. Both types of structure were suggested for several
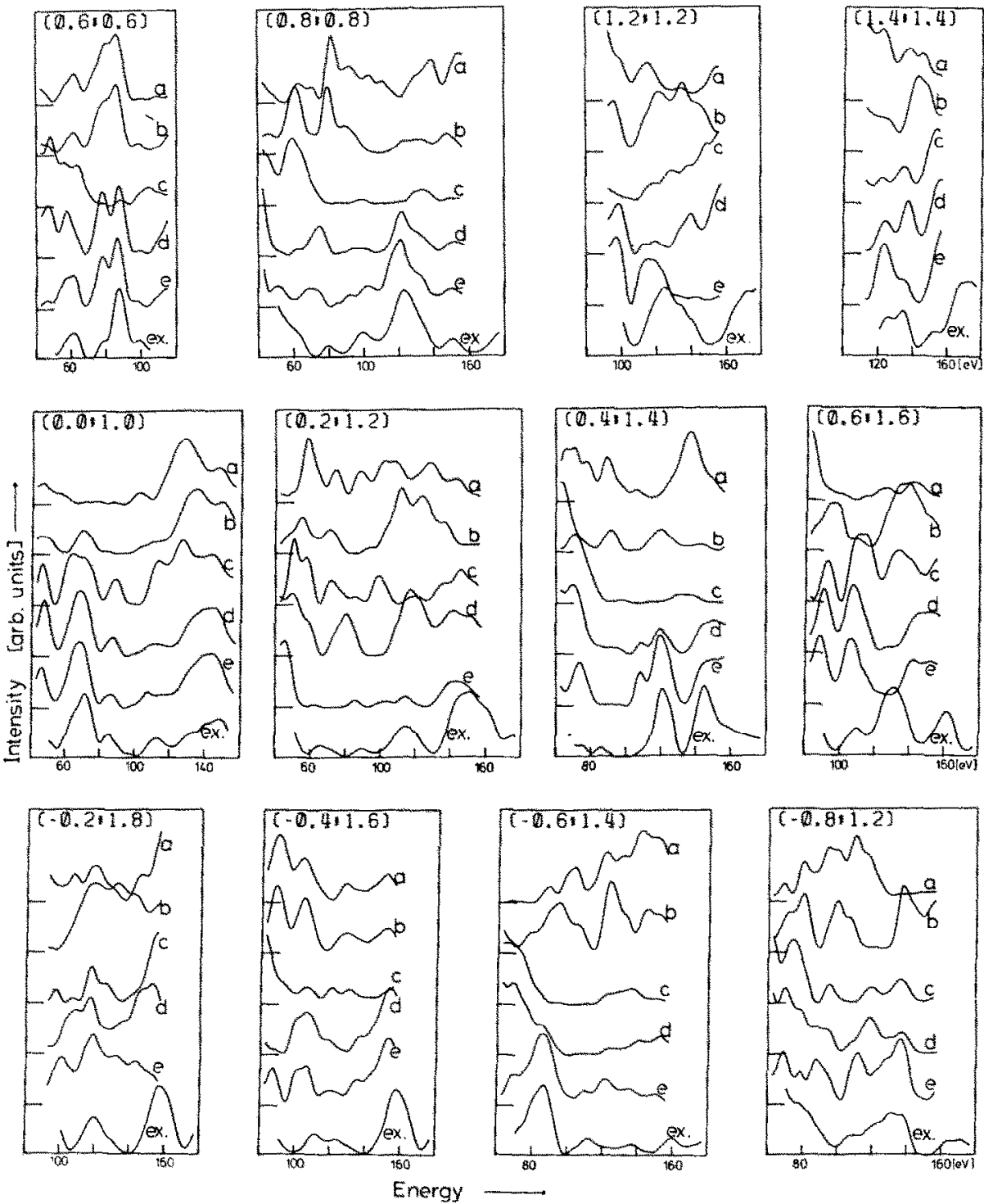

Fig. 4. Comparison between theoretical and experimental spectra of the $c(5 \sqrt{2} \times \sqrt{2}) \mathrm{R} 45^{\circ}$ strueture. The theoretical curves $(a-e)$ were calculated for the five models shown in fig. 3; the layer spacing is $2.4 \AA$; other parameters are as in fig. 2 . 
adsorption systems, none of them has been proved explicitly by LEED calculations.

The models used here are shown in fig. 3. In case of pseudo-hexagonal layers (figs. $3 a$ and $3 b$ ), one lead atom can only be placed in hollow site or top site to maintain the $\mathrm{cmm}$ symmetry of one domain. In case of antiphase models only the hollow site was assumed since these models are derived from the $(\sqrt{2} \times \sqrt{2}) R 45^{\circ}$ structure above. To release the stress of the antiphase boundaries, a slight shift of two $\mathrm{Pb}$ atoms away from the hollow site was considered (figs. $3 \mathrm{~d}$ and $3 \mathrm{e}$ ). To simplify calculations, the lead layer was assumed to be plain, though a buckling seems most probable. In all calculations the top layer distance was varied. The best fit for each model was found for $2.4 \AA$, equal to the value for lead atoms adsorbed in hollow sites. Calculations for all models and experimental data are shown in fig. 4.

Both calculations for quasi-hexagonal arrangements (curves $a$ and $b$ in fig. 4) show nearly no correspondence to experimental curves; the model of all $\mathrm{Pb}$ atoms adsorbed in hollow sites (curve c) also can be excluded. Better results were obtained for the two further models (curves $\mathrm{d}$ and e) with $\mathrm{Pb}$ atoms shifted off from hollow sites at the antiphase boundaries. The same preference also results from a $r$-factor analysis. The minimum $r$-factor values for 14 beams are 0.62 and 0.50 for the hexagonal layers, 0.68 for hollow site adsorption and 0.41 for both models with shifted lead atoms. As above, a visual comparison of the curves gives a clearer preference for the curves $\mathrm{d}$ and e than $r$-factor does.

Though the $r$-factor is poor and obvious discrepancies exist at several beams (for example the $(0.6,1.6)$ beam), we conclude that the structures of figs. $3 \mathrm{~d}$ and $3 \mathrm{e}$ derived from $(\sqrt{2} \times \sqrt{2}) \mathrm{R} 45^{\circ}$ domains, are essentially correct. The $r$-factor of 0.4 should not be taken as the final result of a structure analysis. We expect that more detailed calculations, including buckling of the lead layer and further variations of the lateral shifts, will lead to better results. Furthermore it should be pointed out that the structure may contain four domains, each one of lower symmetry with lead atoms shifted away from mirror planes. If these domains are statistically mixed, the $\mathrm{cmm}$ symmetry of the diffraction pattern is still retained and only the average positions are observablc.

\subsection{Intermediate states}

The coverage range between the $(\sqrt{2} \times \sqrt{2}) \mathrm{R} 45^{\circ}$ and the $\mathrm{c}(5 \sqrt{2} \times \sqrt{2}) \mathrm{R} 45^{\circ}$ structures exhibits diffuse and splitted half order beams (fig. 1). The broadening of beams as well as the satellites appear in every reciprocal mesh in the same manner. This behaviour can be explained by antiphase domains. Moreover the $c(5 \sqrt{2} \times \sqrt{2}) R 45^{\circ}$ structure consists of regularly spaced antiphase boundaries along $[1,1]$, and the streaking and splitting of half order beams thus is due to $(\sqrt{2} \times \sqrt{2}) \mathrm{R} 45^{\circ}$ micro-domains, separated by elements of the $c(5 \sqrt{2} \times$ $\sqrt{2}) \mathrm{R} 45^{\circ}$ structure. The coverage is determined by the ratio of area, both structures occupy. An increase in coverage reduces the $(\sqrt{2} \times \sqrt{2}) \mathrm{R} 45^{\circ}$ area, lessens the distance between domain boundaries and rearranges them. This will 
cause the coverage dependent diffraction patterns in fig. 1. All these structures are disordered, and only the mean features are visible. A detailed study of the diverse domain arrangements will follow.

\section{Summary}

Two ordered structures occurring during deposition of lead on copper (100) have been investigated by LEED intensity measurements and calculations. As expected, the lead atoms of the $(\sqrt{2} \times \sqrt{2}) \mathrm{R} 45^{\circ}$ structure are adsorbed in hollow sites. The results for the $\mathrm{c}(5 \sqrt{2} \times \sqrt{2}) \mathrm{R} 45^{\circ}$ structure indicate that the lead atoms do not form a close packed quasi-hexagonal layer. but maintain nearly fourfold coordination in groups, separated by antiphase boundaries. Each atom at the boundary is shifted by about $0.3 \AA$ off the hollow site. These conclusions are consistent with observations at intermediate coverages, all of which can be explained by arrangements of micro-domains, formed by introducing antiphase boundaries into the $(\sqrt{2} \times \sqrt{2}) \mathrm{R} 45^{\circ}$ structure.

\section{Acknowledgements}

We thank Prof. Dr. H. Jagodzinski for helpful discussions. Financial support from the Deutsche Forschungsgemeinschaft (SFB 128) is gratefully acknowledged.

\section{References}

[1] J.P. Biberian and M. Huber. Surface Sci. 55 (1976) 259.

[2] J.P. Biberian and G.E. Rhead, J. Phys. F3 (1973) 675;

J.P. Biberian, Surface Sci. 74 (1978) 437.

[3] F. Delamare and G.E. Rhead, Surface Sci. 35 (1973) 172.

[4] J. Perdereau and I. Szymerska, Surface Sci. 32 (1972) 247.

[5] J. Henrion and G.E. Rhead, Surface Sci. 29 (1972) 20.

[6] M. Huber and J. Oudar, Surface Sci. 47 (1975) 605.

[7] J.R. Noonan and H.L. Davis, in: Proc. ICSS-4 and ECOSS-3, Cannes, 1980, p. I 100.

[8] D. Chadwick and A.B. Christie, Surface Sci. 82 (1979) L293;

D. Chadwick and A.B. Christie. in: Proc. ICSS-4 and ECOSS-3, Cannes, 1980, p. 423.

[9] A. Sepulveda and G.E. Rhead, Surface Sci. 66 (1977) 436.

[10] J.B. Pendry, Low Energy Electron Diffraction (Academic Press, London, 1974).

[11] K. Schwarz, Theoret. Chim. Acta 34 (1974) 225.

[12] The phase shifts were calculated with a program made available by $K$. Heinz from the University of Erlangen.

[13] E. Zanazzi and F. Jona, Surface Sci. 62 (1977) 61. 